\title{
Inglés para Preescolares: Una Tarea Pendiente en la Formación Inicial Docente
}

Lidia A. Fuentealba*, Andrew P. Philominraj, Bruno E. Ramirez-Muñoz y Nicol A. Quinteros

Departamento de Idiomas, Universidad Católica del Maule, Talca, Chile. (e-mail: Ifuentealba@ucm.cl; andrew@ucm.cl; bramirez@ucm.cl,nicol23qr@gmail.com)

Recibido Ago. 30, 2018; Aceptado Nov. 27, 2018; Versión final Dic. 20, 2018, Publicado Jun. 2019

\begin{abstract}
Resumen
El propósito de este artículo exploratorio fue diagnosticar el conocimiento acumulado en relación a las didácticas de la enseñanza del inglés como lengua extranjera centradas en la edad pre-escolar. A través de una metodología mixta se analizaron 32 artículos publicados en base de datos ERIC, Scielo y Scopus, desde el año 2014 al 2017. Los resultados demuestran que la enseñanza de un idioma extranjero para preescolares es incipiente, y a pesar de que no está formalizado en el currículo nacional preescolar, igualmente se enseña en diferentes comunidades educativas. Desde esta realidad ya existente se genera la necesidad de develar estrategias apropiadas para el trabajo con preescolares, que permitan dotar de nuevas herramientas a los docentes en su formación inicial.
\end{abstract}

Palabras clave: enseñanza preescolar; lengua extranjera; didáctica del inglés; currículo; formación inicial docente

\section{English for Preschoolers: A Pending Task in Initial Teacher Training}

\begin{abstract}
The purpose of this exploratory article was to diagnose the present situation of the teaching of English as a foreign language focused on pre-school age. Through a mixed methodology, 32 articles published in ERIC, Scielo and Scopus databases from 2014 to 2017 were analyzed. The results show that the teaching of a foreign language for preschoolers is incipient, and although it is not formalized in the national preschool curriculum, it is anyway taught in different educational communities. From this existing reality there is a need to unveil the appropriate strategies for working with preschoolers, which will provide new tools for teachers in their initial training.
\end{abstract}

Keywords: preschool teaching; foreign language; English didactics; curriculum; initial teacher training 


\section{INTRODUCCIÓN}

La educación puede entenderse como un proceso de formación y empoderamiento de individuos, creadores de respuestas eficientes a las nuevas condiciones impuestas por la globalización. Esto involucra descubrir y fomentar las capacidades y habilidades de los educadores y los aprendices bajo el concepto de desarrollo holístico para la integración exitosa del individuo hacia la vida y la sociedad (Philominraj et al., 2017). El aprendizaje de un idioma, junto con ser un instrumento social, es también un elemento transversal ya que constituye la plataforma en donde residen valores, actitudes, características culturales y relaciones interpersonales, que conforman la base para la construcción de una sociedad y el aprendizaje (Lomas et al., 2003). El aprendizaje de una lengua extranjera es un tema relevante en los sistemas educativos a nivel nacional e internacional, dado que se percibe que es "una inversión para el futuro que puede producir muchos beneficios tanto a nivel profesional, para propósitos de crecimiento personal o viajes" (Weatherford, 2006). Entre los múltiples beneficios de aprender una lengua extranjera se encuentran la mejora de las habilidades matemáticas (Bathia y Ritche, 2004) y las capacidades analíticas e interpretativas (Bruck, 2007). En este sentido, el idioma inglés se ha transformado en una herramienta de comunicación a nivel mundial, por lo que su enseñanza tanto como segunda lengua o lengua extranjera ha requerido empezar cada vez más temprano en la educación primaria.

Entre los estudios llevados a cabo para documentar las didácticas que se utilizan para enseñar inglés como idioma extranjero (EFL) a preescolares, se destacan el uso de canciones, rimas, cuentos, y tecnología, entre otros. Es decir, la enseñanza de la lengua extranjera responde al gustos y preferencia de los niños (Huete y Morales, 2003). Por consiguiente, el acercarse didácticamente a lo que naturalmente prefieren los niños, facilitaría de cierto modo la motivación e interacción, ya que se les estaría involucrando en actividades sociales y comunicacionales dentro de su propio contexto e intereses acorde a su rango etáreo (Uysal y Yavuz, 2015). Adicionalmente, cabe mencionar que los aspectos socioculturales de la enseñanza de inglés como idioma extranjera no han sido muy explorados; no está muy claro si lo investigado en la enseñanza del inglés como segunda lengua pudiera aplicar en contextos de instrucción y ambiente completamente diferentes (Sun, et al., 2016). Lo cierto es que en el mundo globalizado de hoy, el inglés como idioma extranjero ha llegado a quedarse y está siendo relevante en todos los ámbitos. Dado este escenario, este artículo intenta a dar cuenta de la tarea pendiente en la formación inicial de la enseñanza del inglés como lengua extranjera, con énfasis en la enseñanza preescolar.

\section{Enseñanza del inglés}

La enseñanza del inglés como segunda lengua (ESL) o inglés como lengua extranjera (EFL) son parte de programas educacionales en distintas partes del mundo. De hecho, la literatura da cuenta de diferentes enfoques y metodologías que señalan las técnicas más adecuadas para la enseñanza del inglés como segunda lengua (Richards y Renandya, 2011); sin embargo, no se explicita la diferencia de los enfoques y técnicas aplicadas en un ambiente en que se aprende la lengua como idioma extranjero (Nayar, 1997). Asimismo, la literatura ha develado que la mejor decisión pedagógica de un profesor en cuanto a la enseñanza de una lengua que no es la materna, es a través de la selección de diferentes enfoques y métodos disponibles, para verificar prácticas docentes que hayan sido exitosas (Celce-Murcia, 2001) y situadas de acuerdo a la particularización de cada contexto o escenario para la actividad pedagógica (Kumaravadivelu, 2001). En el caso de América Latina, el inglés se ha ido posicionando a raíz de políticas que tienen como base estrategias económicas relacionadas con los procesos de globalización, por lo que el inglés ha pasado a ser obligatorio desde la enseñanza primaria, y desde la educación preescolar, informalmente. Los países latinoamericanos han diseñado estrategias nacionales para crear programas de aprendizaje del inglés para todos y todas, y para ofrecer oportunidades reales de acceso al aprendizaje de otro idioma (Cronquist y Fiszbein, 2017).

De acuerdo a los resultados de un estudio realizado por Education First (EF), institución dedicada al fomento de la cooperación internacional por medio de la promoción del aprendizaje, Chile muestra bajos resultados a nivel latinoamericano, posicionando al país en el lugar 45 de un total de 80 países, lo cual es bajo al promedio de los países menos desarrollados de América Latina (Education First, 2017). De acuerdo a este índice que evalúa el nivel de competencia del idioma inglés hay una fuerte correlación entre países con mayor distribución equitativa del poder, reducción de la desigualdad social y entornos más democráticos con resultados más altos en el dominio del inglés (Education First, 2018). Estudios realizados en el año 2004 reportan que solo el $10 \%$ de los estudiantes chilenos que ingresan a las Universidades Chilenas son capaces de acreditar un examen de competencia lingüística en el idioma internacional (Cancino, 2007). Según King (2007) se comprueba que los estudiantes egresados de colegios municipalizados y subvencionados poseen un dominio rudimentario de la comprensión de la lengua escrita y los niveles básicos de producción oral, habilidades que forman parte de los objetivos fundamentales del programa de inglés para esta etapa. Junto con lo anterior, de acuerdo a Philominraj (2015), se debe destacar que en 
Chile menos de un $2 \%$ de los adultos trabajadores hablan un nivel moderado de inglés; por lo tanto, el dominio de una segunda lengua o lengua extranjera, podría ser una herramienta de conocimiento que generaría actitudes de respeto, tolerancia y valorización intercultural, contribuyendo no solo al conocimiento lingüístico, sino que también al crecimiento personal y social de la comunidad.

\section{Educación pre-escolar}

La educación primaria es uno de los pilares fundamentales del desarrollo del recurso humano, motor del progreso del país (Delors, 2013). La evidencia muestra que la educación preescolar es beneficiosa, y los niños que participan de ella desarrollan mejores competencias (Melhuish, 2011; Nores y Barnes, 2010). Asimismo, Yeung (2014) expone que la educación temprana tiene una directa relación con el desarrollo físico, social, emocional y cognitivo del niño y tiende a desarrollar de mejor forma sus habilidades sociales y aprendizaje. Es por esto que la enseñanza preescolar ha tomado cada vez más importancia para los sistemas gubernamentales, exponiendo la necesidad de invertir en ella para obtención de beneficios a largo plazo en educación (Barnett, 2008).

La enseñanza del inglés a preescolares es una práctica común en los establecimientos educacionales chilenos, aun cuando no cuenten con los documentos curriculares oficiales que respalden esta praxis; por ende, se hace necesario indagar y entender de qué manera se puede facilitar el aprendizaje de este idioma en este nivel en beneficio de los niños. De acuerdo a Copland y Garton (2014), el aprendizaje del inglés por niñas y niños pequeños ha aumentado considerablemente a través de los años, motivo por el que han aumentado también las investigaciones en este rango etario. De la misma manera, Nikolov, citado en Tekin (2015) establece que es precisamente la primera infancia el periodo más significativo para el desarrollo y aprendizaje del lenguaje incluso cuando exista una exposición limitada a la lengua extranjera. De hecho, la mayoría de las investigaciones han sido desarrolladas por docentes e investigadores que están en la búsqueda de respuestas que den cuenta de la realidad de una exposición restringida a lengua extranjera.

El dominar dos o tres idiomas no es inusual en el mundo de hoy; de hecho, la exposición a instrumentos tecnológicos como videos en línea, hace que los niños estén expuestos a diferentes lenguas, acentos, y modismos. Esta exposición a diferentes variedades dialectales favorece la asimilación de características segmentales y supra segmentales del idioma, lo que promueve "la habilidad fonético-fonológica que se ejercita naturalmente en el aprendizaje temprano del inglés [pues] favorece la menor eliminación de sonidos en el interior de la palabra por la exposición a un segundo idioma (Fuica y Soto-Barba, 2014). De esta misma forma, investigaciones han enfatizado la relación que existe entre el desarrollo de las habilidades de procesamiento fonológico a temprana edad para la adquisición de la capacidad lectora (Stanovich, 1988; Vellutino, Scanlon y Lyon, 2000; Wagner y Torgesen, 1987 citado por Herrera y Defior, 2005). Esta exposición a otro idioma va en la línea de la comunicación en un mundo globalizado, en el que los niños necesitarán comunicarse en, al menos, dos lenguas extranjeras para desenvolverse en el mundo variado y multicultural que se está construyendo.

Investigadores revelan que la educación preescolar puede producir ganancias substanciales en el aprendizaje y desarrollo de los niños (Barnett, 2002) además de abrir diferentes oportunidades, tales como la activación temprana de nuevos intereses y curiosidades y entusiasmo para otros idiomas extranjeros (Pinter, 2017). De igual manera, estudios demuestran que la enseñanza temprana puede mejorar considerablemente el aprendizaje y desarrollo de un niño proveniente de un contexto sociocultural vulnerable (Barnett, 2008). Según Melhuish (2011) las evidencias de que la educación preescolar es beneficiosa es contundente; los niños que asisten a la educación preescolar terminan con mejores resultados en la certificación General de Educación Secundaria (GCSE). Así mismo, estudios internacionales empíricos han comprobado que el aprendizaje de una lengua extranjera a temprana edad permite al niño alcanzar un alto dominio del lenguaje que no alcanzarían aquellos que comienzan a una edad más tardía (Khalifa, 2012).

Científicos de la U. College de Londres han detectado que conocer un segundo idioma modifica positivamente la estructura del cerebro, mejorando su plasticidad e incluso la materia gris es más densa en la parte inferior de la corteza parietal de quienes hablan una segunda lengua, y más aún si lo hacen desde una edad temprana (BrainLang, 2017). Ser bilingüe implica hacer conexiones y relaciones más rápidas en ambos idiomas lo que es posible e debido a que los conceptos están incorporados en el cerebro y facilita el aprendizaje. Un estudio llevado a cabo por Gargía_Pentón y otros (2014) señala diferencias entre hablantes monolingües y bilingües, mostrando estos últimos una mayor conectividad en dos subredes críticas para el procesamiento del lenguaje y el control cognitivo (citado en Li y Grant, 2015). El dominio de la lengua inglesa no beneficiaría solamente aspectos económicos a largo plazo, sino que también sensibilizaría hacia aspectos culturales producto del contacto y conocimiento de otras culturas, ya que la exposición a una lengua diferente a la materna contribuiría a la reflexión y conocimiento de la cultura y lengua propia (Shin y 
Crandall, 2014), puesto que cuando se aprende un segundo idioma se adquiere una segunda mirada del mundo que nos rodea (Kecskes, 2005). Asimismo, la habilidad de producción oral eficiente contribuye al desarrollo temprano de la alfabetización tanto en la lengua materna del niño (Gordon y Browne, 2001) y en su segundo idioma (Yesil- Dagli, 2011). Muchos niños bilingües han evidenciado habilidades metalingüísticas muy desarrolladas (Bialystock, 1997; Garcia, 2000).

\section{METODOLOGÍA}

Este estudio es de carácter exploratorio realizado por medio de la recolección de documentos relacionados al tema en estudio a través de la técnica de investigación cualitativa "bola de nieve" (Creswell, 2009); vale decir, de la referencia bibliográfica de un artículo se obtuvo otros, además, se utilizaron frases claves en la búsqueda de información para dar cuenta de la tarea pendiente en la formación inicial de la enseñanza del inglés como lengua extranjera, con énfasis en la enseñanza preescolar. Documentos rescatados en bases de datos tales como: ERIC, Scielo, Scopus, y Google académico tanto en inglés como en español fueron seleccionados y analizados. Por la naturaleza del tema, la información rescatada en español no era suficiente. Este proceso de recolección de información tuvo dos fases; primero, la clasificación global de todo lo relacionado con el tema, ya fueran tesis, presentaciones académicas en seminarios, ensayos, reseñas, y artículos; de aquella primera recogida, se obtuvo 54 documentos, 37 de ellos en inglés y 17 en español.

Posteriormente, en la segunda fase, de los 54 documentos clasificados, se seleccionó solamente los artículos que tuvieran relación con el tema del estudio. Por lo tanto, el corpus de análisis fue formado por 32 artículos, y un compendio de diferentes estudios publicados en un libro; 8 de ellos escritos en español y 24 en inglés; todos extraídos de bases de datos ERIC, Scielo, y Scopus, entre los años 2014 y 2018, con excepción de cuatro estudios que datan de los años 2003, 2009, 2010, y 2012, que, por la relevancia de la información entregada, fueron incluidos en los estudios a analizar. Los 32 estudios seleccionados y clasificados en año de publicación, país de origen, objetivo de estudio, enfoque de investigación y sugerencias de los autores, representan las inquietudes de docentes e investigadores educativos del área de la enseñanza y aprendizaje de lenguas extranjeras.

\section{RESULTADOS Y DISCUSIÓN}

A partir de la información clasificada se analizaron los estudios de acuerdo a país de origen, año de publicación, objetivo del estudio, y técnicas de investigación. El análisis nos muestra que la enseñanza del inglés como lengua extranjera en diferentes países que no comparten la misma lengua, se ha ido masificando por razones económicas y/o académicas (Shin y Crandall, 2014); lo que evidencia la necesidad de entender las didácticas y estrategias de enseñanza más apropiadas que se puedan aplicar en un contexto de instrucción extranjero. Se puede observar que el número de artículos publicados por docentes e investigadores en el área de las didácticas de enseñanza de inglés como lengua extranjera ha ido en aumento entre los años 2003 al 2018, es decir, se está tratando de dilucidar cuales son las estrategias didácticas más apropiadas de acuerdo a factores tales como la edad, la cultura, estilos de aprendizaje, entre otros. Asimismo, y en relación a la enseñanza del inglés como lengua extranjera a preescolares, se deduce que es un tema incipiente, y que se necesitan más estudios en diferentes contextos para realmente entender la forma en que los niños aprenden una lengua extranjera (Yim, 2016).

La tabla 1 nos muestra el país de origen que indica el lugar en el que se llevó a cabo el estudio, y que de acuerdo a la clasificación, Colombia y España son los países que lideran el número de estudios en ésta área, ya que ambos países cuentan con estrategias nacionales para la enseñanza de lenguas.

Colombia Bilingüe ha adoptado como estrategia nacional mejorar las habilidades de los estudiantes en la lengua inglesa, y de esa forma tributar al movimiento social y económico de los ciudadanos (Cronquist y Fiszbein, 2017). Se requiere construir un universo bilingüe aun cuando éste sea un contexto en el que se habla inglés como lengua extranjera. Dicho objetivo desafía de alguna manera a las didácticas de enseñanza utilizadas para enseñar una lengua, ya que el contexto de instrucción no es bilingüe, y por ende, la escasa o nula necesidad de usar el inglés fuera de un aula hace difícil motivar e involucrar a niños y adolescentes en su aprendizaje (Guevara y Ordoñez, 2012). Por lo tanto, el desarrollo de estudios que señalen las vías más adecuadas para la labor de la enseñanza del inglés en un contexto en el que el inglés no es considerado una segunda lengua, son de beneficio para los docentes y las políticas de país. 
Tabla 1: País de origen

\begin{tabular}{|ll|}
\hline País de origen & Número de publicaciones \\
\hline Colombia & 4 \\
\hline España & 8 \\
\hline México & 1 \\
\hline Costa Rica & 1 \\
\hline Indonesia & 1 \\
\hline Taiwán & 1 \\
\hline Estados Unidos & 3 \\
\hline Corea del Sur & 1 \\
\hline Israel & 1 \\
\hline China & 1 \\
\hline Serbia & 1 \\
\hline Saudí Arabia & 1 \\
\hline Turquía & 4 \\
\hline Japón & 1 \\
\hline Irán & 1 \\
\hline Austria & 1 \\
\hline
\end{tabular}

Tabla 2: Temáticas exploradas en los objetivos de estudio

\begin{tabular}{|lc|}
\hline \multicolumn{1}{|c|}{ Temática } & $\begin{array}{c}\text { Número de } \\
\text { estudios }\end{array}$ \\
\hline Revisión teórica de materiales, pautas, y propuestas para la enseñanza del inglés en niñas y niños & 13 \\
\hline $\begin{array}{l}\text { Impacto y/o beneficio de diferentes didácticas de la enseñanza del inglés como lengua extranjera en } \\
\text { niñas y niños }\end{array}$ & 14 \\
\hline Formación pedagógica de profesores en la enseñanza del inglés en preescolares y educación primaria & 5 \\
\hline
\end{tabular}

Las temáticas exploradas en el total del corpus de análisis pueden clasificarse en tres ejes fundamentales que están relacionados con la teoría y la práctica; clasificación que da cuenta de los actores fundamentales que tributan a la enseñanza aprendizaje de todo educando: La formación de profesores en la especialidad, la revisión teórica de la enseñanza específica en un área, y el impacto o beneficio de la aplicación de diferentes didácticas de la enseñanza en contextos diversos y de acuerdo a diferentes realidades.

El primer eje da cuenta de la revisión teórica de materiales, programas, y propuestas para la enseñanza del inglés en nivel preescolar. De aquella información, se devela la necesidad de contar con las herramientas teóricas y empíricas para la adecuada implementación de un currículo de enseñanza de inglés a niveles locales; de hecho, entre los objetivos de estudios, algunos de ellos ofrecen revisión de literatura específica sobre la educación del inglés entre niños y niñas en algunas partes del mundo. Dicho esto, podemos deducir que la búsqueda de respuestas podría deberse a la creciente demanda de la enseñanza de esta lengua en diferentes comunidades; y también como lo declara Gordon y Browne (2001), la enseñanza del inglés como lengua extranjera a niños pequeños es un área de la enseñanza muy específica, en la que los docentes deben estar conscientes de cómo aprenden los niños, pero también cómo ellos aprenden otra lengua que no es la materna.

El segundo eje abarca el número más alto de estudios recolectados y está íntimamente relacionado al eje anterior ya que, entre los objetivos de estudio clasificados en este eje, se busca determinar, caracterizar, evaluar, y explorar la aplicación de didácticas de enseñanza de inglés como lengua extranjera en preescolares y niveles primarios. Los estudios indican, en su mayoría, que el inglés debería ser enseñado a través de las actividades más acordes a la edad y el contexto en que el niño o niña se encuentre (Sikki et al, 2013), es decir, aquellos estudios buscan develar el escenario más confiable, seguro, y beneficioso para los estudiantes, de acuerdo a contextos físicos, sociales, culturales, y cognitivos, entre otros. Es relevante mencionar que, en la recolección y selección de artículos relacionados a este eje, se incluyó estudios que daban cuenta de didácticas aplicadas en niveles primarios también, ya que la edad en el comienzo de la escolaridad varía de acuerdo a políticas internas de cada país. 
El tercer eje da cuenta de la formación pedagógica del profesorado, de aquellos que trabajan con niños, pero que no tienen la especialidad o experticia de trabajar con pequeños. Por un lado, están los especialistas en niveles preescolares y primarios, pero que no tienen las competencias lingüísticas para enseñar el inglés como lengua extranjera. Por otro lado, están los docentes que tienen las competencias para enseñar otra lengua, pero que no poseen el conocimiento básico para trabajar en niveles preescolares y primarios. Siguiendo esta línea, los objetivos de estudio de algunos de estos artículos buscan analizar la formación pedagógica de los profesionales de la educación inicial, explorar patrones del lenguaje de profesores de diferentes niveles lingüísticos que enseñen inglés como lengua extranjera, investigar las percepciones de aquellos docentes que enseñan inglés en niveles primarios, caracterizar el desarrollo profesional que se ofrece en algunas comunidades educativas, como también exponer a profesores en formación universitaria a micro enseñanzas, para desarrollar en ellos habilidades pedagógicas para enseñar inglés a niños en edad preescolar.

Tabla 3: Enfoque de la investigación

\begin{tabular}{|lc|}
\hline Metodología de investigación & Número de estudios \\
\hline Mixto & 14 \\
\hline Cualitativo & 13 \\
\hline Cuantitativo & 5 \\
\hline
\end{tabular}

Asimismo, como se señala en la tabla 3, existe una tendencia a desarrollar estudios mixtos y cualitativos, y en menor medida, de corte cuantitativo. Si bien no se explicita en los estudios por qué se prefirió un enfoque por sobre el otro, se puede deducir que el enfoque mixto está siendo mayormente usado por la riqueza de la información que se puede recolectar. El enfoque mixto permite complementar miradas y experiencias desde una perspectiva holística del objeto que se requiere analizar (Pereira, 2011).

\section{DISCUSIÓN FINAL}

Las anteriormente planteado coincide en sugerir y destacar la importancia y relevancia de explorar en la enseñanza y aprendizaje del inglés como lengua extranjera en niveles preescolares haciendo referencia a los aspectos teóricos, prácticos y pedagógicos relacionados con los ejes establecidos en este artículo (Copland y Garton, 2014). Los estudios muestran la necesidad de investigar e indagar en vacíos tales como la escasa literatura en estudios relacionados con la materia, la edad que podría influir en el dominio de las habilidades de una lengua extranjera y las actividades apropiadas para enseñar a niños preescolares (Uysal y Yavuz, 2015). Además, es relevante el diseño de material acorde a las necesidades y realidades de aquellos que comienzan el aprendizaje en edad temprana (Aguilar, 2017), así como también el trabajo docente y parental que está detrás del aprendizaje de cada preescolar. También es importante mencionar la necesidad de investigación en relación al rol de los padres o cuidadores de familia. Por lo tanto, el docente debe tener conocimiento sobre el desarrollo social y cognitivo de los niños, así como de las teorías de la enseñanza de una segunda lengua (Copland y Garton, 2014), las cuales deben ser congruentes a la edad y contexto en que se encuentra el educando, y contar con la experiencia necesaria que le haya permitido poner en práctica y adaptar principios lingüísticos y didácticos (Yilorm, 2016), prácticas que entregarán las competencias necesarias para aplicar diferentes metodologías y estrategias de enseñanza que realmente ayuden a los estudiantes a aprender (Sikki et al., 2013).

Cualquiera sea el modelo de enseñanza de una lengua, éste será efectivo si promueve la interacción, la motivación y la actitud positiva hacia el aprendizaje de la lengua (Shin y Crandall, 2014) y considera que el error que comete el alumno es parte normal y funcional del aprendizaje (Villarroel, 1997). Vale decir, las prácticas docentes se deben ir modificando de acuerdo a las necesidades particulares de los diferentes contextos sociales y culturales en los que se está situado. Para la enseñanza del inglés a preescolares se debería generar conocimiento específico que facilite los procesos de enseñanza aprendizaje de este grupo de aprendientes. De acuerdo a Uysal y Yavuz (2015), existe número limitado de estudios relacionados con la enseñanza temprana de una lengua extranjera en vez de una segunda lengua. De la misma manera, existe escasa información sobre las didácticas de la enseñanza para preescolares que sean efectivas en aulas donde los niños tengan input limitado y sean parte de grupos numerosos (Copland y Garton, 2014).

Finalmente, y tal como lo declara Cameron (2003), la enseñanza temprana del inglés a preescolares es una realidad que está creciendo, y que necesita ser desarrollada en el campo de la enseñanza del inglés a hablantes de otras lenguas. Se hace necesario contar con profesionales competentes con habilidades pedagógicas para la enseñanza del inglés a pre-escolares, así como también la exploración de la enseñanza de esta lengua situada en los variados contextos educacionales. 


\section{CONCLUSIONES}

En base a lo antes mencionado, se concluye que la enseñanza-aprendizaje del inglés como lengua extranjera en nivel preescolar es una realidad incipiente que necesita ser indagada por expertos de dos áreas principalmente: de la enseñanza de una lengua extranjera y de la enseñanza específica para niños.

\section{AGRADECIMIENTOS}

Se agradece apoyo del Programa Formación de Capital Humano Avanzado CONICYT / 21182041 y al Proyecto FIC BIP №40.001.166-0

\section{REFERENCIAS}

Aguilar, B.S., La Universidad y su Participación en las Comunidades de Aprendizaje: un compromiso para la transformación social y educativa, doi 10.20420/ElGuiniguada.2017.135, El Guiniguada Revista de investigaciones y experiencias en Ciencias de la Educación, 34-46 (2017)

Barnett, W.S., Early Childhood Education. In Molnar, A., School Reform Proposals: The Research Evidence. 1-6. CT: Information Age Publishing,Greenwich, The United States (2002)

Barnett, W.S., Why Governments Should Invest in Early Education, National Institute for Early Education, 6 (2), (2008)

Bhatia, T. y W. Ritchie, The Handbook of Bilingualism, Malden, Blackwell Pub (2004)

Bialystock, E., Effects of Bilingualism and Biliteracy on Children's Emerging Concepts of Print, doi.org/10.1037/00121649.33.3.429, Developmental Psychology, 33(3), 429-440 (1997)

BrainLang, C., Beneficios de Aprender un Segundo Idioma (2017)

Burck, C., Multilingual Living:Explorations of Languageand Subjectivity, Houndmills, Palgrave Macmillan (2007)

Cameron, L., Challenges for ELT from Expansion in Teaching Children, doi.org/10.1093/elt/57.2.105, ELT Journal, 57(2), 105-112 (2003)

Cancino, N., Aproximaciones a Itaa Enseñanza de las Lenguas en Chile. Trabajo Presentado en el $5^{\circ}$ Congreso Internacional de Cultura y Desarrollo, La Habana, Cuba (2007)

Celce-Murcia, M., Teaching English as a Second or Foreign Language, $3^{\mathrm{a}}$ Ed., 5(4), Heinle y Heinle, Boston, MA (2001)

Copland, F. y S. Garton, Key Themes and Future Directions in Teaching English to Young Learners: Introduction to the Special Issue, doi.org/10.1093/elt/ccu030, ELT Journal, 68(3), 223-230 (2014)

Creswell, J. W., Research Design: Qualitative, Quantitative, and Mixed Methods Approaches, $2^{a}$ Ed., Sage publications, Thousand Oaks, California (2009)

Cronquist, K. y A. Fiszbein, El Aprendizaje del Inglés en América Latina, 86 (2017)

Delors, J., Los Cuatro Pilares de la Educación (2013)

Education First, English Profiency Index (2017)

Education First, English Profiency Index (2010)

Fuica, M. y J. Soto-Barba, Ajustes fonético-fonológicos en niños(as) de 4 a 5 años que aprenden inglés como segunda lengua, https://dx.doi.org/10.4067/S0716-58112014000200014, Literatura y lingüística, (30), 258-283 (2014)

García, G.E., Bilingual Children's Reading. In M.L. Kamil, P. B. Mosenthal, P.D. y R. Barr (Eds.), Handbook of Reading Research, Mahwah, New Jersey, Lawrence Erlbaum Associates, 3, 813-834 (2000)

Gordon, A.M., y K. W. Browne, La Infancia y su Desarrollo, Delmar, Thomson Learning, USA (2001)

Guevara, D.C. y C.L. Ordoñez, Teaching English to very Young Learners through Authentic Communicative Performances, Colombian Applied Linguistics Journal, ISSN: 2248-7085, 14(2), 9-23 (2012)

Herrera, L. y S. Defior, Una Aproximación al Procesamiento Fonológico de los Niños Prelectores: Conciencia Fonológica, Memoria Verbal a Corto Plazo y Denominación, https://dx.doi.org/10.4067/S0718-22282005000200007, Psykhe (Santiago), 14(2), 81-95 (2005)

Huete, C. y V. Morales, Enseñanza-Aprendizaje de las Lenguas Extranjeras en Edades Tempranas, Servicio de Publicación y Estadística, 343 (2003)

Kecskes, I., Cognitive Approaches to Bilingualism: Introduction to the Special Issue, doi.org/10.1177/13670069050090010101, International Journal of Bilingualism, 9 (1), 1-6 (2005) 
Khalifa, E.M., The Effects of Age Factor on Learning English: A Case Study of Learning English in Saudi Schools, Saudi Arabia, doi:10.5539/erl.v5n1, English Language Teaching, 5 (1), 127 (2012)

King, P., Estudio Multidimensional de la Oralidad a partir de los Textos Escolares para la Enseñanza del Inglés como Lengua Extranjera, doi: 10.4067/S0718-09342007000100006, Revista Signos, 40(63), 101-126 (2007)

Kumaravadivelu, B., Toward a Postmethod Pedagogy, Tesol Quarterly, 35, 4, 537 (2002)

Li, P. y A. Grant, Second language learning success revealed by brain networks, doi:10.1017/S1366728915000280, Bilingualism, Language and Cognition, 19, 657-664 (2016)

Lomas, C., A. Osorio y A. Tusón, Ciencias de Lenguaje. Competencia Comunicativa y Enseñanza de la Lengua, Oto Saku, Madrid, España (2003)

Melhuish, E., Education Preschool Matters, doi:10.1126/science.1209459, Science Journal, 333, 299-300 (2011)

Nayar, P.B., ESL/EFL Dichotomy Today: Language Politics or Pragmatics?, doi:10.2307/3587973, TESOL Quarterly, 31(1) (1997)

Nores, M. y W.S. Barnett, Benefits of Early Childhood Interventions across the World:(Uder)Investing in the very Young, Economics of Education Review, 29(2), 271-282 (2010)

Pereira, P.Z., Mixed Method Designs in Education Research: A Particular Experience, Revista Electronica Educare, ISSN: 51409-42-58, 15 (1), 15-29 (2011)

Philominraj, A. y otros autores, Bilingualism - A Challenge for New Horizons, doi: dx.doi.org/10.7813/jll.2017/8-2/1, Journal of Language and Literature, 8(2), 5-11 (2017)

Philominraj, A., An Exploratory Study of the Best Indian ELT Practices for the Chilean ESL Learners, Thesis Doctoral, The Loyola College, University of Madras, India (2015)

Pinter, A., Teaching Young Language Learners, Oxford University Press, London (2017)

Richards, J.C. y W.A. Renandya, Methodology in Language teaching: an Anthology of Current Practice, New York, Cambridge University Press (2011)

Shin, J.K. y J. Crandall, Teaching English to Young Learners around the World: from Theory to Practice (2014)

Sikki, E., A. Rahman, A. Hamra y N. Noni, The Competence of Primary School English Teachers in Indonesia, Journal of Education and Practice, ISSN:222-1735, 4(11), 139-146 (2013)

Sun, H., R. Steinkrauss, J. Tendeiro y K. De Bot, Individual Differences in Very Young Children's English Acquisition in China: Internal and External Factors, doi.org/10.1017/S1366728915000243, Bilingualism: Language and Cognition, 19(3), 550-566 (2016)

Tekin, A.K., Early EFL Education Is on the Rise in Oman: A Qualitative Inquiry of Parental Beliefs about Early EFL Learning, doi:10.5539/elt.v8n2p35, English Language Teaching, 8(2), (2015)

Uysal, N.D. y F. Yavuz, Teaching English to Very Young Learners, doi.org/10.1016/j.sbspro.2015.07.042, Procedia Social and Behavioral Sciences, 197, 19-22 (2015)

Villarroel, M., Persistencia y Cambio en la Enseñanza del Inglés, https://dx.doi.org/10.4067/S0716-58111997001000011, Literatura y lingüística, (10), 205-219 (1997)

Weatherford, H.J., Personal Benefits from Foreign Language Study, Clearinghouse on Languages and Linguistics (1986)

Yesil-Dagli, U., Predicting ELL Students' Beginning First Grade English Oral Reading Fluency from Initial Kindergarten Vocabulary, Letter Naming and Phonological Awareness Skills, Early Childhood Research Quarterly, 26, 15-29 (2011)

Yeung, C., The Importance of Early Childhood Education (2014)

Yilorm, Y., Proceso de Enseñanza Aprendizaje de la Lengua Inglesa en Escuelas Públicas Chilenas: ¿Producción o Reproducción?, doi.org/10.4067/S0718-07052016000300009, Estudios Pedagógicos, 42 (Número Especial), 103-116 (2016)

Yim, S.Y., EFL young learners: Their Imagined Communities and Language Learning, doi.org/10.1093/elt/ccv037, ELT Journal, 70(1), 57-66 (2016) 